\title{
Anestesia libre de opioides en hemicolectomía abierta derecha extendida. Reporte de un caso y revisión
}

\author{
Opiod Free Anesthesia (OFA) for Open extended right \\ colectomy. A case report and review
}

Juan Pablo Ghiringhelli M. ${ }^{1}$, Rodrigo Fuentes², Paula O'Reilly F. ${ }^{1}$

\begin{abstract}
Opioid free anesthesia (OFA) is defined as an anesthesiologic technique where opioids are not used in the intraoperative and postoperative period. Although the mainstay of intra-operative analgesia may be opioids, current challenges are focus on reducing them and preventing the adverse effects of opioids, by rationalizing and even suspending their perioperative use, specifically at risk populations such as Obstructive Sleep Apnea Syndrome (OSAHS), obesity, Chronic Obstructive Pulmonary Disease (COPD) and cancer surgery. We present this case of OFA in a susceptible patient with complications from the use of opioids undergoing an extended right hemicolectomy. Multimodal analgesia was performed with a thoracic peridural and subanesthetic doses of intravenous agents including dexmedetomidine, ketamine and propofol, accompanied by short and long-lasting local periglotic anesthetics. The patient had given an intra- and postoperative analgesia without presenting any adverse events, good recovery, early deambulation and extubation.
\end{abstract}

\section{RESUMEN}

La anestesia libre de opioides (OFA) es una técnica anestésica donde no hay administración de opioides, tanto en el intraoperatorio como en el postoperatorio. Aunque una de las bases de la analgesia intraoperatoria podrían ser los opioides, los desafíos actuales están enfocados en reducir su uso periopera-

\section{Key words:}

Obesity, chronic obstructive behaviour, non-narcotic, obstructive, opioid free, postoperative analgesics, pulmonary disease, sleep apnea, techniques, hemicolectomy

\section{Palabras clave:}

Anestesia libre de opioides, analgésicos opioides,

Profesor Asociado, División de Anestesiología.

2 Residente de Anestesiología Escuela de Medicina, Cátedra de Anestesiología y Reanimación, Universidad de Valparaíso. Viña del Mar, Chile.

Fecha de envío: 04 de febrero de 2020

Fecha de aceptación: 15 de mayo de 2020

\section{ORCID}

https://orcid.org/0000-0002-1909-7661

Correspondencia:

Dr. Juan Pablo Ghiringhelli M. juanpablo.ghiringhelli@uv.cl 
torio, previniendo sus efectos adversos, racionalizando y limitando su empleo específicamente en poblaciones de riesgo como síndrome de apnea obstructiva del sueño (SAHOS), obesidad, enfermedad pulmonar obstructiva crónica (EPOC) y cirugía oncológica. Presentamos este caso de OFA en un paciente susceptible de complicaciones por uso de opioides sometido a una hemicolectomía derecha extendida. Se realizó analgesia multimodal con peridural torácica y dosis subanestésicas de agentes endovenosos como dexmedetomidina, ketamina y propofol, acompañado de anestésicos locales periglóticos de corta y larga duración. Se otorgó una adecuada analgesia intra y postoperatoria, el paciente no tuvo eventos adversos, presentando una buena recuperación, deambulación y extubación precoz. analgésicos,

obesidad,

perioperatorio,

apnea del sueño,

hemicolectomía derecha, cirugía colorrectal

\section{Introducción}

D esde 1960, la administración intraoperatoria de opioides es considerada una piedra angular de la anestesia general moderna, tal como el uso de hipnóticos y relajantes musculares. Actualmente, son la base del tratamiento del dolor intraoperatorio, permitiendo una reducción de la dosis de fármacos inductores, estabilidad hemodinámica y control de la respuesta autonómica al stress quirúrgico[1]. Desde entonces, el paradigma anestésico cambió: de una anestesia inhalatoria a una anestesia multimodal con opioides y bajas dosis de hipnótico. Sin embargo, los objetivos de hipnosis, estabilidad hemodinámica, inmovilidad y analgesia postoperatoria, se pueden lograr sin el uso de opioides[2],[3].

La anestesia libre de opioides (OFA, por sus siglas en inglés), se define como una técnica anestésica donde no hay administración de opioides sistémicos, neuroaxiales o intracavitarios, tanto en el intraopera- torio como en el postoperatorio[4].

El uso de opioides perioperatorios en los pacientes obesos está asociado a un aumento del riesgo de complicaciones, como agravar el SAHOS, la desaturación postoperatoria y provocar náuseas y vómitos postoperatorios (NVPO)[5]. El SAHOS es de elevada prevalencia en este tipo de pacientes y con frecuencia está infradiagnosticado, por lo que resulta esencial buscar la presencia de trastornos respiratorios del sueño y para ello el cuestionario STOP-BANG (Tabla 1) es el de mayor validez en la actualidad[6].

Presentaremos el caso de un paciente anciano y obeso, con antecedentes de EPOC, una alta probabilidad de SAHOS, portador de un cáncer de colon transverso, que fue sometido a una hemicolectomía abierta extendida por laparotomía supra e infraumbililical; en el que se realizó una OFA con bloqueo peridural torácico, ketamina, dexmedetomidina y anestesia periglótica con lidocaína-levobupivacaína para la intubación orotraqueal.

\begin{tabular}{|c|c|c|}
\hline $\begin{array}{l}\text { Acrónimo } \\
\text { STOP-BANG }\end{array}$ & Cuestionario & Puntaje \\
\hline Snoring & $\begin{array}{l}\text { ¿Ronca usted fuerte? (más fuerte que lo que habla o se escucha a través de una puerta } \\
\text { cerrada) }\end{array}$ & 1 \\
\hline Tired & $\begin{array}{l}\text { ¿Se siente usted frecuentemente cansado o somnoliento durante el día? ¿Se queda usted } \\
\text { dormido durante el día? }\end{array}$ & 1 \\
\hline Observed & ¿Lo ha observado alguien dejar de respirar, ahogarse o boquear durante el sueño? & 1 \\
\hline Blood Pressure & ¿Es usted hipertenso o ha recibido tratamiento por presión arterial alta? & 1 \\
\hline BMI & $\mathrm{IMC}>35 \mathrm{~kg} / \mathrm{m}^{2}$ & 1 \\
\hline Age & Edad $>50$ años & 1 \\
\hline Neck & $\begin{array}{l}\text { Circunferencia de cuello (medida alrededor de la manzana de Adán) }>43 \mathrm{~cm} \text { en hombres } \\
0>41 \mathrm{~cm} \text { en mujeres }\end{array}$ & 1 \\
\hline Gender & Género masculino & 1 \\
\hline
\end{tabular}




\section{Caso clínico}

Paciente de 86 años, programado para hemicolectomía abierta extendida por cáncer de colon T3NOMO, estadio IIA. Dentro de sus antecedentes se encuentran: hipertensión arterial, índice de masa corporal (IMC) de 31,9, EPOC tabáquico activo con 15 cigarrillos/día hace 50 años (37,5 paquetes/año), cardiopatía coronaria revascularizada por angioplastía percutánea y usuario de marcapasos por bloqueo auriculoventricular completo. Tiene antecedentes quirúrgicos: apendicectomía, colecistectomía, esternotomía por taponamiento cardíaco posterior a revascularización coronaria y una prostactectomía por vía transvesical. Las anestesias anteriores fueron sin incidentes. Se obtuvo el consentimiento informado anestésico para anestesia mixta, donde se le explicó las ventajas de minimizar o restringir, si era posible, el uso de opioides en el intraoperatorio, a lo que accedió. Pesaba $90 \mathrm{~kg}$ (corregido de $75 \mathrm{~kg}$ ) por lo que se realizó cuestionario STOP BANG, refiriendo 5 puntos, catalogado como alta probabilidad de SAHOS. Tenía capacidad funcional I y en la ecocardiografía preoperatoria de hace 4 meses presentaba buena función sistólica global y segmentaria con una fracción de eyección del ventrículo izquierdo (FEVI) de $69 \%$.

A su llegada a pabellón se monitorizó en forma estándar agregándose la profundidad anestésica mediante índice biespectral (BIS) y la relajación muscular con monitor TOF-watch. Posteriormente, en posición sentada y bajo técnica aséptica, se realiza punción peridural torácica a nivel T10-T11. El tiempo de instalación fue de $22 \mathrm{~min}$. Luego de eso se procede a la preoxigenación por 3 min a volumen corriente, inducción con Ketodex (ketamina 50 mg y dexmedetomidina $50 \mathrm{ug}$ ), sulfato magnesio $2,5 \mathrm{~g}$, lidocaína 20 $\mathrm{mg}$, propofol $100 \mathrm{mg}$, rocuronio $50 \mathrm{mg}$ todos por vía endovenosa. Al momento de la intubación se realiza analgesia periglótica con instilación de 10 cc con lidocaína $1 \%$ y levobupivacaína 0,25\% por 20 segundos, logrando lo intubación sin incidentes ni cambios en la monitorización. Se inició el mantenimiento de la hipnosis con desflurano ajustando su end-tidal según los valores de BIS y se instaló línea arterial radial derecha. El mantenimiento de la relajación neuromuscular profunda se realizó administrando bolos de rocuronio. De manera concomitante recibe bolos de levobupivacaína al 0,25\% a través del catéter peridural para atenuar la respuesta al estrés quirúrgico, manteniendo la presión arterial media con bolos de fenilefrina o efedrina. También recibió metamizol $2 \mathrm{~g}$, paracetamol 1 $\mathrm{g}$, dexametasona $4 \mathrm{mg}$, ácido tranexámico $1 \mathrm{~g}$ y antibioticoterapia asociada (cefotaxima-metronidazol).
Se realiza laparotomía supra e infraumbilical, donde no se evidencia enfermedad diseminada. Se identifica tatuaje a nivel de colon transverso, realizándose sección en cuña de segmento de colon con al menos $5 \mathrm{~cm}$ de margen de cada lado. Se realizó anastomosis lateroterminal sin incidentes. Tiempo quirúrgico de 88 min. Previo a la extubación recibió 8 puff de bromuro de ipatropio, sugammadex $400 \mathrm{mg}$ y bolo de levobupivacaína 0,125\% 8 cc, extubándose sin incidentes a los 3 min de suspensión del desfluorano. El tiempo anestésico total fue de $131 \mathrm{~min}$, prolongado por la instalación dificultosa de la línea arterial previo a la incisión. Por sus antecedentes se realizó monitorización postoperatoria en la Unidad de Cuidados Intensivos $(\mathrm{UCl})$, constatándose al ingreso una $\mathrm{SatO}_{2}$ de $98 \%$ con $\mathrm{FiO}_{2} 50 \%$, frecuencia cardiaca de $61 \times$ min, presión arterial 93/52 $\mathrm{mmHg}$, dolor por Escala Visual Análoga (EVA) de 0. Evolucionó favorablemente, con buen control del dolor con analgesia peridural con Lbupivacaína $0,1 \%$ controlada por el paciente (PCA), sin episodios de desaturación, buena tolerancia oral y herida operatoria sin signos de complicación, siendo trasladado a sala al segundo día postoperatorio. Se mantuvo con analgesia peridural durante las primeras $48 \mathrm{~h}$, siendo evaluado diariamente por el equipo del dolor. El registro de EVA e infusión peridural se detallan en la Figura 1. El promedio de bolos demandados diarios fue de 8 y administrados 3 , indicándose retiro de la peridural a las $72 \mathrm{~h}$.

Inició alimentación a las $24 \mathrm{~h}$ postoperatorias con buena tolerancia, con inicio de tránsito intestinal a las $56 \mathrm{~h}$ postoperatorias. Inició deambulación a las $18 \mathrm{~h}$ postoperatorias. El tiempo de estadía hospitalaria fue de 9 días.

\section{Discusión}

Presentamos este caso de OFA, de manejo multimodal, basándose en una analgesia peridural torácica, con la interacción de dosis subanestésicas de diversos agentes endovenosos como la dexmedetomidina, ketamina y propofol, acompañado de anestésicos locales periglóticos de corta y larga duración.

La administración perioperatoria de opioides está asociada con náuseas y vómitos postoperatorios (PONV), sedación, íleo, confusión/delirium, prurito, retención urinaria, inmunosupresión, alteración cicatrización, hiperalgesia, dolor crónico postoperatorio, hipoxemia, depresión respiratoria y aumento de consumo de morfina postoperatorio[4],[7],[8]. Estos efectos adversos son la principal justificación del porqué los opioides no están incluidos o se encuentran mini- 


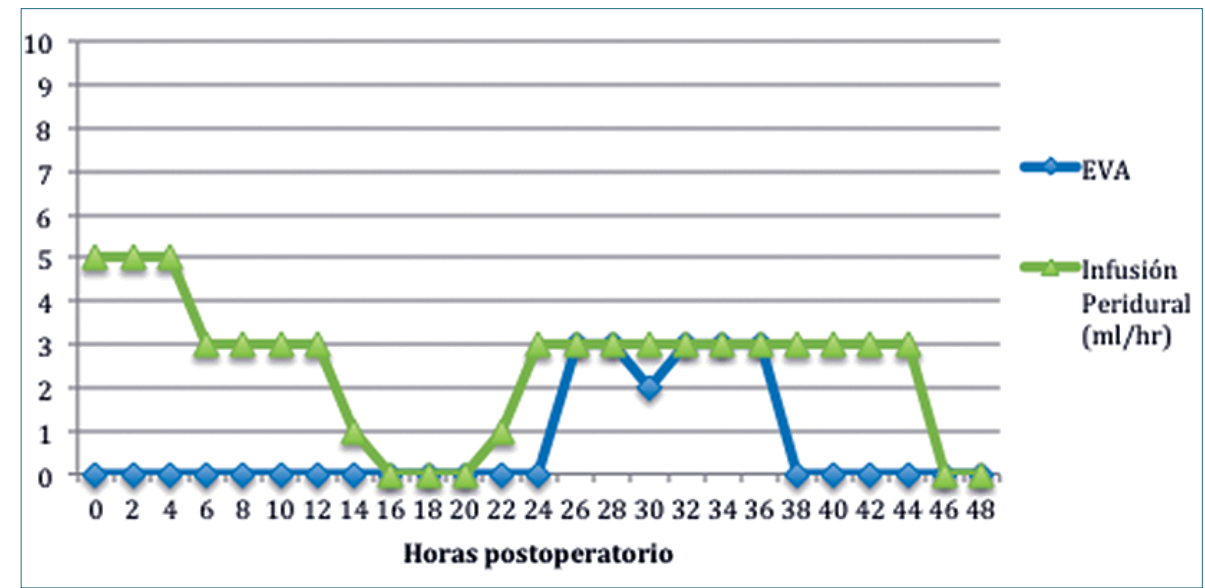

Figura 1. Evaluación dolor postoperatorio a $48 \mathrm{~h}$.

Tabla 2. Indicaciones y ventajas de anestesia libre de opioides (OFA)[4]

\begin{tabular}{ll}
\hline Indicaciones & Ventajas \\
Obesidad & - Condiciones equivalentes intraoperatorias a los opioides \\
SAHOS & - Analgesia con menos efectos adversos \\
EPOC & - Disminución PONV, íleo e hipoxemia, tolerancia e hiperalgesia \\
Adicción y dependencia opioides & - Disminución tiempo en recuperación y estadía hospitalaria \\
Alergia a opioides & - Disminución dolor crónico postquirúrgico \\
Síndrome dolor regional complejo & - Menor riesgo de sobredosis, abuso, uso indebido de recetas y adicción \\
Cirugía oncológica & \\
\hline
\end{tabular}

mizados en los protocolos ERAS[9], donde los pacientes tratados con opioides tienen mayor estadía hospitalaria, aumentando los costos[10]. Si bien es cierto que la OFA produce inicialmente desconcierto y resistencia al cambio; surgiendo preguntas como: "¿para qué se usa?, ¿los pacientes tienen dolor?, ¿para qué complicarse?"; la OFA es posible en la práctica anestesiológica diaria, evitando los principales efectos adversos de los opioides y otorgando una anestesia general segura y estable. Más aún, es una alternativa recomendable en cierto grupo de pacientes como obesos, ancianos, portadores de SAHOS, EPOC, síndrome dolor regional complejo, alergia y adicción a opioides y cirugía oncológica[4]. Así, los reportes de OFA han aumentado considerablemente desde 1980 a la fecha[11]. Los pacientes que se benefician particularmente de una OFA y las ventajas de ésta se especifican en la Tabla 2.

La técnica recomendada y más usada para una hemicolectomía abierta extendida es una anestesia mixta[12], con uso de opioides tanto en forma sistémica como neuroaxial. Este paciente obeso, anciano, EPOC y probablemente con SAHOS no diagnosticado ni tratado, era más susceptible a las complicaciones de los opiáceos, especialmente a la depresión respiratoria producida por su uso. Además, la cirugía era oncológica, donde si bien aún está en materia de discusión, se cree que el uso de morfina promovería el crecimiento del cáncer ya que el receptor de crecimiento tumoral es coactivado por los receptores $\mu[13]$. El paciente se extubó precozmente, presentando buena recuperación sin delirium, íleo ni complicaciones pulmonares postoperatorias.

La OFA -estrictamente- es una combinación de diversas estrategias ahorradoras de opioides, culminando en la completa eliminación del uso de opiáceos, como el uso de antagonistas del receptor glutamaérgico N-metil-d-aspartato (NMDA): ketamina, sulfato de magnesio, corticoides, antiinflamatorios, betabloqueadores, alfa-2-agonistas (clonidina, dex- 
medetomidina); además de anestésicos locales vía endovenosa, tópica, bloqueo neuroaxial y bloqueo de nervio periférico[4]. Aunque cada intervención por sí sola disminuye el uso de opiáceos, la combinación de ellas no necesariamente tendrá un efecto aditivo o sinérgico. Las técnicas utilizadas en este caso fueron:

- Ketamina: Es el único inductor anestésico con propiedades analgésicas, por su antagonismo NMDA, teniendo un rol fundamental en el manejo del dolor postoperatorio al reducir el fenómeno de wind up y la sensibilización central. Previene la liberación de potasio desde la célula y la consiguiente transmisión del impulso doloroso. Su uso como dosis única, incluso a dosis pequeñas como 0,1 mg/kg en el perioperatorio, disminuye los requerimientos de morfina en las primeras 24 h postoperatorias[14],[15].

- Dexmedetomidina: Sus efectos farmacológicos se condicen con los pilares de la anestesia libre de opioides: hipnosis, sedación, analgesia y simpaticolisis. Como fármaco simpaticolítico, permite minimizar la respuesta simpática al estrés generado por la intubación traqueal y mantener un patrón hemodinámico estable, siendo su principal efecto adverso la bradicardia y en menor medida la hipotensión[16]. Se ha incluido como uno de los fármacos principales de la OFA en pacientes obesos, con SAHOS y/o EPOC, reduciendo significativamente el dolor postoperatorio y en casi un $40 \%$ el consumo de opioides sin causar depresión respiratoria en el paciente obeso[17],[18]. La combinación de ketamina y dexmedetomidina en la misma jeringa en bolo (ketodex), es clínicamente efectiva y posee un buen perfil de estabilidad, no observándose cambios en la FC y presión arterial como si pudiesen ocurrir administrando cada una por separado[19].

- Anestesia periglótica o anestesia tópica de la vía aérea: la anestesia tópica con ropivacaína previo a la intubación reduce las fluctuaciones hemodinámicas de la intubación e incluso permite mejores condiciones de intubación en ausencia de relajo muscular. Además, disminuye significativamente la tos en la extubación y la odinofagia postoperatoria[20]. En este caso se ocuparon anestésicos locales de corta y larga duración (lidocaína y Ibupivacaína), presentando buena recuperación postoperatoria, sin tos ni odinofagia. Aún no hay consenso sobre su incorporación como práctica en la OFA, principalmente por la falta de estudios de mayor escala para evaluar la anestesia tópica en el ahorro de opioides perioperatorio. No obstante, la lidocaína endovenosa, sea en bolo o infusión, es una de las técnicas más usadas y validadas en la OFA, por sus propiedades analgésicas y antiinflamatorias, disminuyendo el consumo de opiáceos en el postoperatorio[4].

- Anestesia peridural torácica: el bloqueo simpático neuroaxial se constituye en este caso como un pilar fundamental en la OFA. Su uso en grandes cirugías abdominales, como laparotomías medias supra e infraumbilicales, otorga significativamente mejor control del dolor postoperatorio en las primeras dos semanas, supresión de la respuesta quirúrgica, permitiendo tiempos de extubación más rápidos y disminuyendo los requerimientos de opiáceos y analgésicos en el período postoperatorio[21],[22]. De manera concomitante en ancianos y en cirugía de colon presenta un beneficio adicional, presentando mayor satisfacción del paciente, aumento del flujo sanguíneo colónico, menor incidencia disfunción cognitiva postoperatoria y recuperación más precoz de la función intestinal[12].

- Sulfato de magnesio: El magnesio actúa como antagonista no competitivo NMDA y tiene un efecto antiinflamatorio, disminuyendo los niveles de interleuquina 6 y factor de necrosis tumoral alfa en el postoperatorio. La administración perioperatoria en dosis única $30 \mathrm{mg} / \mathrm{kg}$ tiene un efecto ahorrador de morfina en el tratamiento del dolor postoperatorio de cirugía abdominal[4].

- Otros componentes de analgesia multimodal (metamizol, paracetamol y dexametasona). El enfoque multimodal con estos medicamentos, disminuye los requerimientos de morfina y la estadía hospitalaria, siendo imprescindible en los protocolos de recuperación acelerada despues de cirugía o ERAS[23],[24],[25].

La epidemia de abuso de opioides podría ser una realidad según reportes internacionales, aumentando el número de muertes secundario a sobredosis de opioides. Asimismo, el número de prescripciones de opioides se ha cuadruplicado entre 1999 y 2015 y el acceso ilegal a ellos también[26]. Esta "crisis opioide" se debe en parte a la ambulatorización de las cirugías, entregando opioides orales al domicilio con un control no óptimo. Recientemente algunos han cuestionado la responsabilidad del anestesiólogo en la prescripción de opioides en el intra y postoperatorio para el manejo del dolor quirúrgico en pacientes con adicción y abuso de opiáceos[27]. Ya en 2017, Brummett y cols, reportó una tasa de abuso de opioides postoperatorio de $6 \%$ en 36.177 pacientes quirúrgicos, concluyendo que el uso de opioides luego 
de un procedimiento quirúrgico podría contribuir a una potencial adicción[28]. En Chile, estas cifras están subestimadas, con tasas de abuso de opioides del $0,1 \%[29]$. Incluso el más reciente estudio nacional de drogas publicado el año 2013, no incluía cifras con respecto al abuso de opiáceos[30].

\section{Conclusión}

Presentamos este caso exitoso de OFA en una hemicolectomía derecha extendida en un paciente susceptible de complicaciones por uso de opiáceos donde 1) se otorgó una adecuada analgesia intra y postoperatoria; 2) No tuvo eventos adversos; 3) Bue- na recuperación, deambulación y extubación tempranas. La OFA es posible en la práctica anestesiológica diaria siendo una alternativa cuando se quiere reducir el uso perioperatorio de opioides en pacientes susceptibles de complicaciones por su uso, como lo era el caso clínico. Resulta fundamental contar con estudios multicéntricos de OFA que permitan agrupar un mayor número de pacientes para ratificar sus ventajas y así poder hacer una recomendación basada en evidencia de mejor calidad, principalmente en cuanto a resultados como disminución del dolor crónico posquirúrgico y recurrencia de cáncer. No obstante, las experiencias y reporte de casos individuales constituyen un punto de partida para el eventual desarrollo de estudios a mayor escala.

\section{Referencias}

1. Fawcett WJ, Jones CN. Bespoke intra-operative anaesthesia - the end of the formulaic approach? Anaesthesia. 2018 Sep;73(9):1062-6. https:// doi.org/10.1111/anae.14253 PMID:29533468

2. Hofer RE, Sprung J, Sarr MG, Wedel DJ. Anesthesia for a patient with morbid obesity using dexmedetomidine without narcotics. Can J Anaesth. 2005 Feb;52(2):176-80. https://doi. org/10.1007/BF03027725 PMID:15684259

3. Díaz-Crespo J, Malo-Manso A, Bustamante-Domínguez C, Escalona-Belmonte JJ, Cruz-Mañas J, Guerrero-Orriach JL. Laparotomía en un paciente bajo anestesia libre de opiáceos. An Sist Sanit Navar. 2018;29;41(2):259-62. [Acceso 1 de diciembre de 2019] Disponible en: http://scielo. isciii.es/pdf/asisna/v41n2/11376627-asisna-41-02-259.pdf

4. Sultana A, Torres D, Schumann R. Special indications for Opioid Free Anaesthesia and Analgesia, patient and procedure related: including obesity, sleep apnoea, chronic obstructive pulmonary disease, complex regional pain syndromes, opioid addiction and cancer surgery. Best Pract Res Clin Anaesthesiol. 2017 Dec;31(4):547-60. https://doi. org/10.1016/j.bpa.2017.11.002 PMID:29739543

5. Mulier JP. Perioperative opioids aggravate obstructive breathing in sleep apnea syndrome: mechanisms and alternative anesthesia strategies. Curr Opin Anaesthesiol. 2016 Feb;29(1):129-33. https://doi.org/10.1097/ ACO.0000000000000281 PMID:26595546

6. Nazar C, Coloma R, Contreras J, Molina I, Fuentes R. Consideraciones perioperatorias en el paciente obeso. Rev. Cirugía. 2018;70(6). [Internet], [Acceso 14 diciembre 2019] Disponible en: https://www.revistacirugia.cl/ index.php/revistacirugia/article/ view/6

7. Lee LA, Caplan RA, Stephens LS, Posner KL, Terman GW, Voepel-Lewis T, et al. Postoperative opioid-induced respiratory depression: a closed claims analysis. Anesthesio- logy. 2015 Mar;122(3):659-

65. https://doi.org/10.1097/

ALN.0000000000000564

PMID:25536092

8. Apfel CC, Läärä E, Koivuranta M, Greim CA, Roewer N. A simplified risk score for predicting postoperative nausea and vomiting: conclusions from cross-validations between two centers. Anesthesiology. 1999 Sep;91(3):693-700. https:// doi.org/10.1097/00000542199909000-00022 PMID:10485781

9. Feldheiser A, Aziz O, Baldini G, Cox BP, Fearon KC, Feldman LS, et al. Enhanced Recovery After Surgery (ERAS) for gastrointestinal surgery, part 2: consensus statement for anaesthesia practice. Acta Anaesthesiol Scand. 2016 Mar;60(3):289334. https://doi.org/10.1111/ aas.12651 PMID:26514824

10. Oderda GM, Evans RS, Lloyd J, Lipman A, Chen C, Ashburn $M$, et al. Cost of opioid-related adverse drug events in surgical patients. J Pain Symptom Manage. 2003 Mar;25(3):27683. https://doi.org/10.1016/ S0885-3924(02)00691-7 
PMID:12614962

11. Lavand'homme P, Estebe JP. Opioid-free anesthesia: a different regard to anesthesia practice. Curr Opin Anaesthesiol. 2018 Oct;31(5):556-61. https://doi.org/10.1097/ AC0.0000000000000632 PMID:29994942

12. Patel S, Lutz JM, Panchagnula U, Bansal S. Anesthesia and perioperative management of colorectal surgical patients - A clinical review (Part 1). J Anaesthesiol Clin Pharmacol. 2012 Apr;28(2):162-71. https://doi. org/10.4103/0970-9185.94831 PMID:22557737

13. Sacerdote P, Franchi S, Panerai AE. Non-analgesic effects of opioids: mechanisms and potential clinical relevance of opioid-induced immunodepression. Curr Pharm Des. 2012;18(37):6034-42. https:// do.2174/138161212803582496 PMID: 22747543 https://doi./10. 2174/138161212803582496.

14. Brinck EC, Tiippana E, Heesen M, Bell RF, Straube S, Moore RA, et al. Perioperative intravenous ketamine for acute postoperative pain in adults. Cochrane Database Syst Rev. 2018 Dec;12(12):CD012033. https:// doi.org/10.1002/14651858. CD012033.pub4 PMID:30570761

15. García-Navia JT, Tornero López J, Egea-Guerrero JJ, Vilches Arenas A, Vásquez Gutiérrez T. Effect of a single dose of lidocaine and ketamine on intraoperative opioids requirements in patients undergoing elective gynecological laparotomies under general anesthesia. A randomized, placebo controlled pilot study. Farm Hosp 2016; 40(1):44-51. [Internet], [Acceso 12 diciembre 2019] Disponible en http://manu. edu.mk/prilozi/39_2_3/14.pdf

16. Vaswani JP, Debata D, Vyas V,
Pattil S. Comparative study of the effect of dexmedetomidine vs fentanyl on haemodynamic response in patients undergoing elective laparoscopic surgery. J Clin Diagn Res. 2017 Sep;11(9):UC04-08. https://doi.org/10.7860/ JCDR/2017/27020.10578 PMID:29207810

17. Capasso R, Rosa T, Tsou DY, Nekhendzy V, Drover D, Collins J, et al. Variable Findings for Drug-Induced Sleep Endoscopy in Obstructive Sleep Apnea with Propofol versus Dexmedetomidine. Otolaryngol Head Neck Surg. 2016 Apr;154(4):765-70. Available from: https://doi. rg/10.1177/0194599815625972 https://doi.

rg/10.1177/0194599815625972 PMID:26814208

18. Blaudszun $G$, Lysakowski C, Elia N, Tramèr MR. Effect of perioperative systemic $\alpha 2$ agonists on postoperative morphine consumption and pain intensity: systematic review and meta-analysis of randomized controlled trials. Anesthesiology. 2012 Jun;116(6):131222. https://doi.org/10.1097/ ALN.0b013e31825681cb PMID:22546966

19. Bali KB, Patel A. Study of KETODEX; Combination of Dexmedetomidine and Ketamine in Upper Gastrointestinal Scopy in Adults: observational Study. J Med Sci Clin Res. 2017;05(06):23986-93. https://doi.org/10.18535/jmscr/ v5i6.196.

20. Fang $\mathrm{P}$, Zong Z, Lu Y, Han X, Liu $X$. Effect of topical ropivacaine on the response to endotracheal tube during emergence from general anesthesia: a prospective randomized double-blind controlled study. BMC Anesthesiol. 2018 Sep;18(1):134. https://doi. org/10.1186/s12871-018-0601-x PMID:30261837
21. Freise $H$, Van Aken HK. Risks and benefits of thoracic epidural anaesthesia. Br J Anaesth. 2011 Dec;107(6):859-68. https:// doi.org/10.1093/bja/aer339 PMID:22058144

22. Bello M, Oger S, Bedon-Carte S, Vielstadte C, Leo F, Zaouter $C$, et al. Effect of opioid-free anaesthesia on postoperative epidural ropivacaine requirement after thoracic surgery: A retrospective unmatched case-control study. Anaesth Crit Care Pain Med. 2019 Oct;38(5):499505. https://doi.org/10.1016/j. accpm.2019.01.013 PMID:30731138

23. Arslan M, Celep B, Ciçek R, Kalender HÜ, Yılmaz H. Comparing the efficacy of preemptive intravenous paracetamol on the reducing effect of opioid usage in cholecystectomy. J Res Med Sci. 2013 Mar;18(3):172-7. PMID:23930110

24. De Oliveira GS Jr, Almeida MD, Benzon HT, McCarthy RJ. Perioperative single dose systemic dexamethasone for postoperative pain: a meta-analysis of randomized controlled trials. Anesthesiology. 2011 Sep;115(3):57588. https://doi.org/10.1097/ ALN.0b013e31822a24c2 PMID:21799397

25. Toleska M, Kuzmanovska B, Kartalov A, Shosholcheva M, Nancheva J, Dimitrovski A, et al. Opioid Free Anesthesia for Laparotomic Hemicolectomy: A Case Report. Prilozi (Makedon Akad Nauk Umet Odd Med Nauki). 2018 Dec;39(2-3):121-6. https://doi.org/10.2478/prilozi-2018-0050 PMID:30864360

26. Boysen PG 2nd, Pappas MM, Evans B. An Evidence-Based Opioid-Free Anesthetic Technique to Manage Perioperative and Periprocedural Pain. Ochsner J. 2018;18(2):121-5. https:// 
doi.org/10.31486/toj.17.0072

PMID:30258291

27. Steyaert A, Lavand'homme P. Postoperative opioids: let us take responsibility for the possible consequences. Eur J Anaesthesiol. 2013 Feb;30(2):50-

2. https://doi.org/10.1097/

EJA.0b013e32835b9db2

PMID:23303242
28. Brummett CM, Waljee JF, Goesling J, Moser S, Lin P, Englesbe MJ, et al. New Persistent Opioid Use After Minor and Major Surgical Procedures in US Adults. JAMA Surg. 2017 Jun; 152(6):e170504. https://doi.org/10.1001/jamasurg.2017.0504 PMID:28403427

29. SENDA, Décimo estudio nacional de drogas en población general, 2012, 2013, Ministerio del Interior y Seguridad Pública Gobierno de Chile.

30. CONACE. Séptimo estudio nacional de drogas en población general, 2006, 2007, Ministerio del Interior y Seguridad Pública Gobierno de Chile, Santiago de Chile. 